\title{
The Siberian multimodal brain tumor image segmentation dataset
}

\author{
Sergey Golushko \\ Novosibirsk State University \\ Novosibirsk, Russia \\ s.k.golushko@gmail.com \\ Evgeniya Amelina \\ SDA\&ML lab. \\ Novosibirsk State University \\ Novosibirsk, Russia \\ amelina.evgenia@gmail.com \\ Vladimir Groza \\ Median Technologies \\ Valbonne, France \\ vladimir.groza@gmail.com
}

\author{
Mikhail Amelin \\ Radiodiagnosis division \\ ORCID: 0000-0002-5099-5144 \\ FSBI "Federal Neurosurgical Center" \\ Novosibirsk, Russia \\ amelin81@gmail.com \\ Nikolay Tolstokulakov \\ SDA\&ML lab. \\ Novosibirsk State University \\ Novosibirsk, Russia \\ n.tolstokulakov@g.nsu.ru
}

\author{
Bair Tuchinov \\ SDA\&ML lab \\ Novosibirsk State University \\ Novosibirsk, Russia \\ bairts@gmail.com \\ Evgeniy Pavlovskiy \\ SDA\&ML lab. \\ Novosibirsk State University \\ Novosibirsk, Russia \\ pavlovskiy@post.nsu.ru
}

\begin{abstract}
Automatic brain tumor segmentation from CT or MRI scans is one of the crucial problems among other directions and domains where daily clinical workflow requires to put a lot of efforts while studying patients with various pathologies. In this paper, we report the results of the research project "Brain Tumor Segmentation" organized in conjunction with the Federal Neurosurgical Center. Several state-of-the-art tumor segmentation algorithms were applied to a set of 100 MRI scans of meningioma, neurinoma and glioma patients - manually annotated by up to three raters - and to 100 comparable scans obtained using the automated tumor multi-region segmentation. Quantitative evaluations revealed a considerable agreement between the human raters in segmenting various tumor subregions (Dice scores in the range $85-90 \%$ ). We found that different algorithms worked best for different sub-regions, but no single algorithm ranked in the top for all subregions simultaneously.
\end{abstract}

Keywords - Neural Network, Deep Learning, Segmentation, Medical Imaging

\section{INTRODUCTION}

Brain tumors are usually very heterogeneuous by its structure and edema is simillar to the healthy or non-enhanced tumor tissues. Within the recent achievements the problem of segmentation of the brain tumors can be effectively solved with the use of 2D and 3D deep learning (DL) based methods. However, none of structural magnetic resonance imaging (MRI) such as T1, T2, contrast-enhanced T1 (T1-CE), and T2 Fluid Attenuated Inversion Recovery (FLAIR) sequences or other permits to observe and identify all tumor composition elements by eyes or with the use of existing (semi-) automated techniques. Using the thorough analysis one could detect and classify diseased tissue and different pathological processes such as edema, necrosis, tumor etc. It is well known that being a crucial task for the effective diagnostics and differentiation between pathological processes the proper segmentation is also a source of recommendations for further treatment and followup.

\section{METHODS}

We used several approaches and data processing techniques. In the core of this framework we use the "LinkNet- like" networks with two different backbones from SeResnext50 and Se-Resnext101. Additionally we tried methods including following techniques: mixture of T1, T1-CE, and T2 FLAIR sequences in a pseudo-RGB image; combination of three T1C images as another pseudo-RGB image including the neighboring slices; wide input where all three sequences are used to create 9-channel input images and others. In order to compare obtained results we define the baseline method using only the T1C images, and also studying the difference between standard ResNet networks and its shift-invariant version with the blur levels.

\section{RESULTS}

Results presented in this work were obtained on the private dataset contained 100 full brain scans - manually annotated by up to three raters, 100 comparable scans obtained using the automated tumor multi-region segmentation. Annotations comprise labels of the peritumoral edema, the non-enhancing tumor, GD-enhancing tumor and the necrotic tumor core. We set-up the prototype the Siberian Brain Tumor Image Segmentation dataset (SBT). Quantitative evaluations revealed a considerable agreement between the human raters in segmenting various tumor sub- regions (Dice scores in the range $85-90 \%$ ). We found that different algorithms worked best for different sub-regions, but that no single algorithm ranked in the top for all subregions simultaneously.

\section{ACKNOWLEDGMENT}

The reported study was funded by RFBR according to the research project No. 19-29-01103.

\section{REFERENCES}

[1] Kamnitsas, K., Bai, W., Ferrante, E., McDonagh, S., Sinclair, M., et al. Ensembles of Multiple Models and Architectures for Robust Brain Tumour Segmentation. MICCAI Brainlesion Workshop. pp. 450-462. Springer, 2017.

[2] Myronenko, A. 3D MRI Brain Tumor Segmentation using Autoencoder Regularization. MICCAI Brainlesion Workshop. pp. 311-320. Springer, 2018.

[3] Letyagin, A. Y., Golushko, S. K. et al. Artificial Intel- ligence for Imaging Diagnostics in Neurosurgery. In 2019 International MultiConference on Engineering, Computer and Information Sciences (SIBIRCON) (pp. 336-337). IEEE-INST. ELECTRICAL ELECTRON - ICS ENGINEERS INC. 